\title{
The Catalase Gene Differentiates between Some Strains of Staphylococcus aureus ssp. anaerobius
}

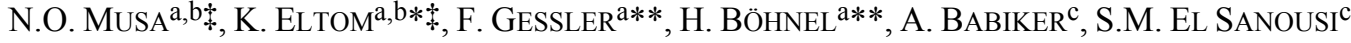 \\ anstitute of Tropical Animal Health, Georg-August University of Göttingen, D-37077 Göttingen, Germany \\ ${ }^{\mathrm{b}}$ Institute for Promotion of Animal Export Studies, University of Khartoum, 13314 Shambat, Khartoum North, Sudan \\ ${ }^{\mathrm{c}}$ Faculty of Veterinary Medicine, University of Khartoum, 13314 Shambat, Khartoum North, Sudan
}

\begin{abstract}
Staphylococcus aureus ssp anaerobius strain S10 was isolated from an outbreak of sheep abscess disease. Sequence of the catalase gene of this strain showed $99 \%$ identity to the catalase gene $(\mathrm{katB})$ sequence of the reference strain (S. aureus ssp. anaerobius strain MVF213) with mismatching of three base pairs. An important substitution located 1036 nucleotides upstream of the initiation codon from " $\mathrm{C}$ " in katB to " $\mathrm{T}$ " in the catalase gene of strain S10 originated a stop codon. The deduced protein (345 amino acids) is 105 amino acids shorter than that of katB. Partial sequence of the catalase gene of other 8 local isolates in addition to another reference strain (DSM 20714/ATCC 35844) revealed the same mutations in all local (African) strains, whereas the sequence of the reference (European) strain was typical to that of katB. Sequence of the catalase gene of S. aureus ssp. anaerobius strain S10 was deposited in GenBank under accession no. EU281993.
\end{abstract}

Abbreviations

katA catalase gene of Staphylococcus aureus

katB catalase-like protein gene of Staphylococcus aureus ssp. anaerobius MVF213

katS10 catalase-like protein gene of Staphylococcus aureus ssp. anaerobius strain S10

SaanS10 Staphylococcus aureus ssp. anaerobius strain S10

Anaerobic Staphylococcus aureus bacteria are the causal agent of sheep abscess or Morel's disease (Bajmócy et al. 1984; Hamad et al. 1992). Although these bacteria are considered nonpathogenic for humans, a report on a case of septicemia due to one strain of these bacteria in man has recently been published (Peake et al. 2006). These bacteria were separated from other $S$. aureus bacteria in a subspecies (S. aureus ssp. anaerobius) because of their negative or weak growth in normal air, lack of the catalase activity in addition to some other biochemical properties (de la Fuente et al. 1985). Although some strains of S. aureus ssp. aureus were reported to lack this catalase enzyme activity (Tu et al. 1976; Friedberg et al. 2003; Yilmaz et al. 2005), they still grow well under aerobic conditions (Grüner et al. 2007).

Comparative studies between the catalase genes of $S$. aureus ssp. aureus and S. aureus ssp. anaerobius (katA and katB, respectively) showed that katA had undergone mutations leading to deletion of one base pair in addition to 8 silent and 6 mis-sense mutations (Sanz et al. 2000). The deletion resulted in shift of the reading frame and premature termination of translation with subsequent generation of $k a t B$, which codes for a protein 55 amino acid residues shorter than katA. Lack of the catalase activity of $S$. aureus ssp. anaerobius is attributed to some of these mutations (Sanz et al. 2000). The loss of catalase activity in some strains of S. aureus ssp. aureus was also attributed to mutations of the catalase gene $(\mathrm{katA})$. While in a methicillin-resis$\operatorname{tant} S$. aureus ssp. aureus strain deletion of five successive base pairs led to a shift in the reading frame and premature termination of translation (Grüner et al. 2007), substitution of a key amino acid in the protein (His58Tyr) led to inactivity of this gene in methicillin-sensitive S. aureus ssp. aureus strain (Piau et al. 2008).

The aim of this investigation was to characterize by molecular means some $S$. aureus ssp. anaerobius strains isolated from animals. We conducted full sequencing of the catalase gene of one isolate from an outbreak of sheep abscess disease in addition to partial sequencing of this gene for nine other isolates including one reference strain. We report here on some strains of $S$. aureus ssp. anaerobius harboring a catalase gene that underwent mutations other than those previously reported for the European strains.

*Corresponding author; Institute for Promotion of Animal Export Studies, University of Khartoum, 13314 Shambat, Khartoum North, Sudan; fax +249185326827, e-mail keltom@daad-alumni.de.

**Present address: Institute of Applied Biotechnology in the Tropics at Georg-August University of Göttingen, Marie-Curie-Str. 7, 37079 Göttingen, Germany.

\$These authors contributed equally to the paper. 


\section{MATERIALS AND METHODS}

Bacterial strains. S. aureus ssp. anaerobius strain S10 (SaanS10) was isolated from superficial lymph node abscess of one lamb in a flock of sheep during outbreak of Morel's disease in Alsamra village (East Nile Province of the Sudan) (Musa et al. 2007). Other isolates and strains used were: two isolates from animals in the same disease outbreak, 6 isolates from superficial lymph node abscesses of sheep at meat inspection in abattoirs located in two different areas of Khartoum State, and S. aureus ssp. anaerobius DSM 20714/ ATCC 35844 as a reference strain. Identification of the isolates was based on a failure of aerobic growth within $2 \mathrm{~d}$, lack of catalase activity, positive coagulase activity, in addition to the fermentation ability of some sugars. All tests were done according to standard methods (Barrow and Feltham 1993).

DNA extraction. Genomic DNA was extracted using Axy Prep Bacterial Genomic DNA Miniprep Kit of Axygen (Bioron, Germany) with some modifications of the manufacturer's protocol. In brief, 3-5 colonies from a 2-d blood agar culture were suspended in $150 \mu \mathrm{L}$ of the recommended buffer. Lysis of the cells was achieved by treatment with $10 \mu \mathrm{L}$ of $1 \%$ lysostaphin (Sigma, Germany) for $1 \mathrm{~h}$ at $37{ }^{\circ} \mathrm{C}$ followed, after addition of $2 \mu \mathrm{L} 10 \%$ Proteinase K (Bioron), by a 2 -h treatment at $56{ }^{\circ} \mathrm{C}$. The follow-up steps were carried out according to the manufacturer's protocol.

$P C R$. To confirm the biochemical identification of the isolates, a conserved region of the thermonuclease gene (nuc gene) of $S$. aureus was amplified by PCR using primers and conditions described by Brakstad et al. (1992).

Sequencing of the catalase gene. In order to amplify and sequence the whole catalase gene of SaanS10 and to partially sequence the catalase gene of the other isolates, primers and conditions previously described for the amplification of katA and katB (Sanz et al. 2000) in addition to other primers designed for this purpose were used (Table I). Sequencing was done by Seqlab (Göttingen, Germany). For confirmation of the sequence results, both strands were sequenced, or overlapping parts of the gene were sequenced. Sequences were edited using a software program (BioEdit, Version 7.0.5.3). Alignment and comparisons were done using the Basic Local Alignment Search Tool (BLAST) of NCBI. The resulting sequence of the catalase gene of SaanS10 was deposited in the GenBank under accession no. EU281993; those of the other 8 local isolates and the reference strain were deposited under accession nos FJ935782-FJ935790.

Table I. Oligonucleotides used

\begin{tabular}{|llcc|}
\hline Primer & Sequence $\left(5^{\prime} \rightarrow 3^{\prime}\right)$ & Gene & Source \\
\hline Nuc F & GCG ATt GAT GGT GAT ACG GTT & thermonuclease & Brakstad et al. $(1992)$ \\
Nuc $\mathrm{R}$ & AGC CAA GCC TTG ACG AAC TAA AGC & ditto & ditto \\
$3 \mathrm{~F}$ & GCT TTT TAA GTG TAC TAT TC & catalase & this study $^{\mathrm{a}}$ \\
$164 \mathrm{~F}$ & TAT AAA TTG TGG AGG GAT GAC & ditto & Sanz et al. $(2000)$ \\
$8 \mathrm{~F}$ & CTC CAT TTT AGA ACG CAA CAA & ditto & ditto \\
$1396 \mathrm{~F}$ & GAT GGA TAC GGC TAT GA TA & ditto & this study \\
$872 \mathrm{R}$ & GCT ATA ATT TCA GCA GCT TC & ditto & ditto \\
$1583 \mathrm{R}$ & TGG GTC AGC TTT GTA ACA & ditto & Sanz et al. $(2000)$ \\
$1726 \mathrm{R}$ & TCA TAA ACT GCT CAA CTA CGC & ditto & ditto \\
\hline
\end{tabular}

${ }^{\mathrm{a}}$ The primers were designed based on the sequences of the catalase genes of Staphylococcus aureus strain MVF213 (GenBank accession no. AJ000471) and S. aureus strain ATCC 12600 (GenBank accession no. AJ000472).

\section{RESULTS AND DISCUSSION}

Identification of the isolates by biochemical tests was confirmed by PCR amplification of the nuclease and catalase genes to the species level only (i.e. S. aureus) but not to the subspecies level. Further genetic characterization could be made by sequencing the catalase gene. Sequence of the putative catalase gene of $S$. aureus ssp. anaerobius strain S10 (SaanS10) showed $99 \%$ identity to katB gene of $S$. aureus ssp. anaerobius MVF213 (GenBank accession no. AJ000471), katA gene of $S$. aureus ssp. aureus strains NCTC 8325 and Newman (GenBank accession nos CP000253 and AP009351.1, respectively) and some other strains. The whole amplified part of the putative catalase gene of SaanS10 (katS10) was 1725 nucleotides in length. Comparison of this sequence with $k a t B$ sequence revealed mismatches of only three bases. Fifteen base substitutions occurred within the coding region, when compared with katA, six of which were missense mutations while the others were silent mutations. An important substitution occurred at position no. 1099 
(1036 bases upstream of the initiation codon) of katS10 gene. In katS10 the base is "T", while in katA and $k a t B$ it is "C". This substitution resulted in the code "TGA" instead of "CGA". This code for termination of translation rendered the predicted protein to be only 345 amino acids in length. In $S$. aureus ssp. aureus (NCTC 8325 and Newman strains) the protein of katA is 505 amino acids long. In S. aureus ssp. anaerobius strain MVF213, which is catalase negative, the catalase-like protein of katB is predicted to have 445 amino acids. The loss of the catalase activity of $S$. aureus ssp. anaerobius is attributed to deletion of one base 1338 nucleotides upstream to the initiation codon, which resulted in shift in the reading frame and premature termination of translation 30 bases later (Sanz et al. 2000). In katS10 this deletion is absent, a feature of similarity to katA. The third mismatching of katS10 and katB is that the substitution which occurred at base 949 upstream of the initiation codon leading to serine in katB instead of proline in katA (Sanz et al. 2000) did not happen in katS10. Interestingly, all mutations, except the above mentioned ones, occurred in kat $A$ leading to the generation of $k a t B$ are similar to those mutations associated with katS10. This suggests that katA underwent mutations in at least two steps leading to the generation of katB and katS10.

To investigate for the presence of those mutations of katB and katS10 in other S. aureus ssp. anaerobius isolates, partial sequences (lengths of $\approx 600$ and $\approx 990 \mathrm{bp}$ ) of the catalase genes of 8 other isolates in addition to a reference strain (S. aureus ssp. anaerobius DSM 20714/ATCC 35844) were performed. The segment of the gene chosen for this partial sequence targeted a region that contained most of the mutations seen in katB and katS10, especially those at positions 1503 and 1099, respectively. The sequence of the catalase gene of all local isolates was identical to that of katS10, while that of the reference strain was identical to $k a t B$ sequence.

S. aureus ssp. anaerobius strain MVF213 was originally isolated from a lamb suffering from abscess disease in Spain. The mutations found in this strain leading to the generation of katB were also found in three other strains isolated from lambs affected by the same disease in Spain in different years (Sanz et al. 2000). The Spanish strains thus seem to have originated from one clone (European clone), and the local strains harboring katS10 seem to originate from another genetically distinct clone (African clone). This assumption can be augmented by the results of Elhaj and El Sanousi (2005) who found that local isolates of S. aureus ssp. anaerobius were identical, but distinct from the reference strain, in the DNA restriction pattern in PFGE.

In conclusion, this study shows clear distinction between local and reference strains of $S$. aureus ssp. anaerobius on the basis of the catalase gene sequence. Moreover, our results show that the catalase gene sequence can be used to describe development of regionally specific clones of $S$. aureus ssp. anaerobius. Use of the catalase gene as gene marker for typing strains of $S$. aureus ssp. anaerobius for epidemiological investigations could be further substantiated by studies that will utilize a greater array of isolates from various outbreaks in different geographic sites.

Dr. M.O. Elhaj (Central Veterinary Research Laboratory, Soba, Sudan) provided the reference strain. This work was partially funded by University of Khartoum, Sudan, and by Institute of Applied Biotechnology in the Tropics (IBT) at the University of Göttingen, Germany.

\section{REFERENCES}

BAJMÓCY E., FAZEKAS B., TANYI J.: An outbreak of Morel's disease (a contagious sheep disease accompanied by abscess formation) in Hungary. Acta Vet.Hung. 32, 9-13 (1984).

Barrow G.L., Feltham R.K.A. (Eds): Cowan and Steel's Manual for the Identification of Medical Bacteria, 3rd ed. Cambridge University Press, Cambridge (UK) 1993.

Brakstad O.G., AASBAKK K., MAELAND J.A.: Detection of Staphylococcus aureus by polymerase chain reaction amplification of the nuc gene. J.Clin.Microbiol. 30, 1654-1660 (1992).

Elhaj M.O., El SANOUSi S.M.: Pulsed-field gel electrophoresis for comparison of Staphylococcus aureus subsp. anaerobius local Sudanese isolates. J.Anim.Vet.Adv. 4, 706-707 (2005).

Friedberg B., Hauer E., Belkhirat M., Watine J., Le Coustumier A.: Catalase negative Staphylococcus aureus: a rare cause of catheter-related bacteremia. Clin.Microbiol.Infect. 9, 1253-1255 (2003).

DE LA FuENTE R., SUAREZ G., SCHLEIFER K.H.: Staphylococcus aureus subsp. anaerobius nov., the causal agent of abscess disease of sheep. Internat.J.Syst.Bacteriol. 35, 99-102 (1985).

GrÜNER B.M., HAN S.-R., MeYer H.-G., Wulf U., BhaKDI S., SiEgEL E.K.: Characterization of a catalase-negative methicillin-resistant Staphylococcus aureus strain. J.Clin.Microbiol. 45, 2684-2685 (2007).

Hamad A.R.A.R., Shigiddi M.T., El SAnousi S.M.: Abscess disease of sheep in the Sudan. Sudan.J.Vet.Sci.Anim.Husb. 31, 60-61 (1992).

Musa N.O., Babiker A., El SAnousi S.M., Eltom K.: Outbreak of Morel's disease in the Sudan. Abstract, p. 30 in Tropentag, Witzenhausen, Germany (2007).

PeAke S.L., Peter J.V., Chan L., Wise R.P., Butcher A.R., Grove D.I.: First report of septicemia caused by an obligately anaerobic Staphylococcus aureus infection in a human. J.Clin.Microbiol. 44, 2311-2313 (2006). 
Piau C., Jehan J., LeClerCQ R., DaURel C.: Catalase-negative Staphylococcus aureus strain with point mutations in the katA gene. J.Clin.Microbiol. 46, 2060-2061 (2008).

SAnz R., MARin I., Ruiz-SAnTA-Quiteria J.A., Orden J.A., Cid D., Diez R.M., Silhadi K.S., Amils R., DE LA FuENTE R.: Catalase deficiency in Staphylococcus aureus subsp. anaerobius is associated with natural loss of function mutations within the structural gene. Microbiology 146, 465-475 (2000).

TU K.K., PALUTKE W.A.: Isolation and characterization of a catalase-negative strain of Staphylococcus aureus. J.Clin.Microbiol. 3, 7778 (1976).

Yilmaz M., Aygun G., UtKu T., Dilkmen Y., OztuRK R.: First report of catalase-negative methicillin-resistant Staphylococcus aureus sepsis. J.Hosp.Infect. 60, 188-189 (2005). 